\title{
Dietary acid load, trabecular bone integrity, and mineral density in an ageing population: the Rotterdam study
}

\author{
E. A. L. de Jonge ${ }^{1,2}$ - F. Koromani ${ }^{1,2}$ - A. Hofman ${ }^{1,3}$ - A. G. Uitterlinden ${ }^{1,2}$. \\ O. H. Franco ${ }^{1}$ - F. Rivadeneira ${ }^{2}$ - J. C. Kiefte-de Jong ${ }^{1,4,5}$
}

Received: 20 October 2016 / Accepted: 31 March 2017/Published online: 12 April 2017

(C) The Author(s) 2017. This article is an open access publication

\begin{abstract}
Summary We studied the relation between a diet that is high in acid-forming nutrients (e.g. proteins) and low in base-forming nutrients (e.g. potassium) and bone structure. We showed a negative relation, which was more prominent if proteins were of animal rather than of vegetable origin and if intake of dietary fibre was high.

Introduction Studies on dietary acid load (DAL) and fractures have shown inconsistent results. Associations between DAL, bone mineral density (BMD) and trabecular bone integrity might play a role in these inconsistencies and might be influenced by renal function and dietary fibre intake. Therefore, our aim was to study (1) associations of DAL with BMD and with the trabecular bone score (TBS) and (2) the potential influence of renal function and dietary fibre in these associations.
\end{abstract}

Electronic supplementary material The online version of this article (doi:10.1007/s00198-017-4037-9) contains supplementary material, which is available to authorized users.

J. C. Kiefte-de Jong

j.c.kiefte-dejong@erasmusmc.nl

1 Department of Epidemiology, Erasmus Medical University Centre, Rotterdam, The Netherlands

2 Department of Internal Medicine, Erasmus Medical University Centre, Rotterdam, The Netherlands

3 Department of Epidemiology, Harvard T.H. Chan School of Public Health, Boston, MA, USA

4 Department of Global Public Health, Leiden University College, The Hague, The Netherlands

5 Department of Paediatrics, Erasmus MC, Rotterdam, The Netherlands
Methods Dutch individuals aged 45 years and over $(n=4672)$ participating in the prospective cohort of the Rotterdam study were included. Based on food frequency questionnaires, three indices of DAL were calculated: the net endogenous acid production (NEAP) and the ratios of vegetable or animal protein and potassium (VegPro/K and AnPro/K). Data on lumbar spinal TBS and BMD were derived from dual-energy X-ray absorptiometry measurements.

Results Independent of confounders, NEAP and AnPro/K, but not VegPro/K, were associated with low TBS (standardized $\beta(95 \%)=-0.04(-0.07,-0.01)$ and $-0.08(-0.11$, $-0.04)$ ) but not with BMD. Associations of AnPro/K and VegPro/K with TBS were non-linear and differently shaped. Unfavourable associations between NEAP, BMD and TBS were mainly present in subgroups with high fibre intake. Conclusions High NEAP was associated with low TBS. Associations of AnPro/K and VegPro/K and TBS were nonlinear and differently shaped. No significant associations of DAL with BMD were observed, nor was there any significant interaction between DAL and renal function. Mainly in participants with high intake of dietary fibre, DAL might be detrimental to bone.

Keywords BMD $\cdot$ Dietary acid load · Dietary fibre $\cdot$ Renal function - Trabecular bone score

\section{Introduction}

Fractures are a major health concern in the ageing population and can result in disability and reduced quality of life [1]. Whereas bone mineral density is the most extensively studied determinant of fracture risk, other factors including microdamage, mineralization, bone turnover, macro-geometry of the cortical bone and micro-architecture of the trabecular bone 
are important determinants as well [2]. A novel measure to assess micro-architecture is trabecular bone score (TBS). In brief, this measure combines information on connectivity density, trabecular separation and trabecular number in a single score [2]. In Canadian postmenopausal women, spinal TBS was shown to predict osteoporotic fractures as did hip BMD, but their use in combination incrementally improved prediction [3]. Moreover, spinal TBS was shown to be associated with prevalent and incident vertebral fractures independently of BMD in Dutch participants of the Rotterdam study [4]. Therefore, TBS might be a relevant measure of trabecular bone integrity to study in relation to modifiable lifestyle factors, such as dietary intake.

High dietary acid load (DAL) reflects a diet which is rich in nutrients that are metabolized to non-carbonic acids (e.g. sulphuric acid from the metabolism of protein) in amounts that exceed the quantities of alkali bicarbonate produced from combustion of organic salts (such as potassium chloride in vegetables [5]). Therefore, long-term consumption of such a diet might disturb the balance between $\mathrm{CO}_{2}$ and $\mathrm{HCO}_{3}{ }^{-}$in blood and cause mild but chronic systemic acidosis [6]. DAL has been suggested to affect bone because bone might serve as the primary buffering system for alkali components such as calcium and potassium in case of systemic acidosis [7], but this hypothesis has been contradicted by others [8]. Studies on the relation between DAL and vertebral fractures have shown inconsistent results, and potential associations between DAL and fracture risk are suggested to be mediated by differences in BMD $[9,10]$. That implies that DAL could influence fracture risk via influencing BMD. However, the role of TBS in this association is unclear.

On the one hand, by increasing DAL, dietary protein might have catabolic effects on bone. On the other hand, since the amino acids are important substrates for building bone matrix [11], dietary protein has anabolic effects. It could therefore be hypothesised that associations between DAL and bone outcomes are non-linear.

Whereas the lungs are the primary organs used to neutralize acute metabolic acidosis, chronic disturbances of the acidbase balance are mainly regulated by the kidneys [12]. Renal function is an essential determinant of the regulation of acidbase balance via bicarbonate resorption and acid secretion. Impaired renal function is associated with disturbances in mineral and bone metabolism [13] and fracture risk [7, 14]. For that reason, we hypothesise that participants with altered renal function are less able to maintain a proper acid-base balance when consuming a diet with high acid load and are therefore more likely to develop low BMD and TBS.

Different food groups are known contributors to DAL. Protein sources such as meat, dairy and grain products contribute to a high DAL, whereas sources of potassium such as vegetables contribute to a low DAL. It has been suggested that contrasting associations between DAL and bone outcomes might have been influenced by dietary fibre intake [15]. More specifically, high intake of grains contribute to high DAL and high fibre intake, whereas high intake of vegetables contribute to low DAL and high fibre intake. As dietary fibre might reduce intestinal calcium absorption [15], it could be argued that associations between DAL and bone outcomes might be more detrimental to bone in subjects with high intake of dietary fibre.

Therefore, our main aim was to study the associations of dietary acid load (DAL) with bone mineral density (BMD) and trabecular bone integrity (reflected by TBS) in middleaged and elderly subjects of the Rotterdam study. Moreover, we explored potential non-linear associations. A secondary aim was to assess whether the magnitude of the associations differ according to renal function and intake of dietary fibres.

\section{Methods}

\section{Study design}

This cross-sectional analysis was embedded in the Rotterdam study (RS), a prospective, population-based cohort study. Subjects were middle-aged and elderly people $(n=4672$, SFig. 1) from three RS cohorts. The design and objectives of this study have been described extensively elsewhere [16]. In brief, participating males and females were 45 years or older at the start of the study (1989-1993 for the first cohort (RS-I), 2000-2001 for the second cohort (RS-II) and 2006-2008 for the third cohort (RS-III)). The Rotterdam Study has been approved by the Medical Ethics Committee of the Erasmus MC and by the Ministry of Health, Welfare and Sport of the Netherlands, implementing the Wet Bevolkingsonderzoek: ERGO (Population Studies Act: Rotterdam Study). All participants provided written informed consent to participate in the study and for the study to obtain information from their treating physicians.

\section{Assessment of dietary intake and DAL}

Dietary intake was assessed using a validated, semiquantitative food frequency questionnaire (FFQ) on 389 food items $[17,18]$ at the fifth visit of RS-I (2009-2011), the third visit of RS-II (2011-2012) and the second visit of RS-III (2012-2014). Next, the intake of macro- and micro-nutrients was calculated using the Dutch Food Composition Database (NEVO) from 2006 [19].The net rate of endogenous noncarbonic acid production (NEAP) is a common measure of DAL which is based on the ratio of dietary protein to potassium. This ratio was shown to explain $71 \%$ of the variation in steady-state rate of renal net acid excretion, measured as the sum of the excretion rates of titratable acids and ammonium minus that of bicarbonate previously [5]. In our study, NEAP 
was calculated based on the following equation NEAP $(\mathrm{mEq} /$ day $)=54.4 \times$ protein intake $(\mathrm{g} /$ day $) /$ potassium $(\mathrm{mEq} /$ day $)-$ 10.2 [5]. Subsequently, NEAP was adjusted for total energy intake using the residual method [20]. To study whether acid load due to high vegetable or animal protein intake is differently associated with bone outcomes, protein potassium ratios were calculated using energy-adjusted vegetable protein (for VegPro/K) and animal protein (for AnPro/K) in $\mathrm{mg} /$ day as the numerator and energy-adjusted potassium $(\mathrm{K})$ intake in $\mathrm{mEq} /$ day as the denominator. Two Dutch adult study populations $[17,18]$ have shown that the FFQ was validated to properly rank subjects with respect to high or low intake of nutrients. Pearson's correlation coefficients were 0.62 for calcium and 0.71 for potassium and ranged from 0.59 to 0.68 for total, vegetable, and animal protein after adjustment for total energy intake and sex [17].

\section{Assessment of spinal TBS and BMD}

Lumbar spine (L1-L4) BMD was measured using dual-energy X-ray absorptiometry (DXA) (Prodigy, GE Lunar Corp, Madison, WI, USA). TBS was analysed using TBS iNsight software (Med-Imaps, Geneva, Switzerland) and BMD using GE Lunar software. In brief, TBS is a novel grey-level texture measurement, extracted from DXA images, which correlates with $3 \mathrm{D}$ parameters of bone micro-architecture, connectivity density, trabecular separation and trabecular number. For each region of measurement, TBS was evaluated based on greylevel analysis of the DXA images as the slope at the origin of the log-log representation of the experimental variogram. The method of TBS assessment has been described in detail elsewhere [3].

\section{Assessment of covariates}

Body weight $(\mathrm{kg})$ and height $(\mathrm{cm})$ were measured at the research centre as the subjects wore light clothing and no shoes. Smoking was coded as "current", "past", or "never". Physical activity was estimated using the total number of sports and practising any sport at a professional level based on the validated LASA questionnaire [21]. Plasma vitamin D (25Hydroxyvitamin D in nmol/1) was assessed using electrochemiluminescence immunoassay (COBAS, Roche Diagnostics GmbH, Germany). Estimated glomerular filtration rate (eGFR in $\mathrm{ml} / \mathrm{min}$ ), reflecting renal function, was based on both creatinine and cystatin $\mathrm{C}$. Net household income and highest education attained were categorized into "low", "medium", and "high" as a proxy for socioeconomic status. Dummy variables using the medium category as the reference were used for further analyses. Prevalence of type 2 diabetes was based on fasting glucose $(>11 \mathrm{mmol} / \mathrm{l})$ or use of antidiabetic mediation. Use of lipid lowering or antihypertensive drugs was collected during the home interview. Intake of alcohol, dietary calcium and fibre (in grammes per day), and information on dietary supplement use were assessed using the FFQ. "Supplement use" was defined as taking calcium, vitamin D or multivitamin supplements at least once per week, as these nutrients are components of the DAL and/or important for bone health. Menopausal status was assessed using STRAW-criteria [22]. Use of female hormones was collected using questionnaires and coded as "ever" or "never". All covariates were assessed at the baseline visit of our study, similar to the TBS and BMD data.

\section{Statistical analysis}

To determine which food groups were the main determinants of each of the DAL measures, stepwise backwards regression was used ( $P$ for exclusion $>0.01$ ). Associations of DAL with BMD, TBS were explored using linear regression modelling with NEAP, VegPro/K or AnPro/K as the exposure and TBS, $\mathrm{BMD}$ and presence or absence of vertebral fracture as the outcome (all in study-population specific Z-scores). Subjects with extreme total energy intake $(<500 \mathrm{kcal}$ per day or more than $5000 \mathrm{kcal}$ per day) were removed from the analyses. All analyses were adjusted for age, sex, total energy intake, body weight and height in a basic model (model 1). Based on literature [10] and previous analyses on diet and bone in the Rotterdam study [9, 23], a second model was developed which was further adjusted for smoking, physical activity, socioeconomic status, use of lipid lowering drugs, use of dietary supplements and intake of alcohol and intake of calcium (model 2). Natural cubic splines were computed to explore potential non-linear associations in our most adjusted models (model 2), of which the degrees of freedom were determined based on the lowest Akaike's information criterion (AIC) value. Likelihood ratio tests (LRTs) were performed to determine whether a non-linear model fitted the data significantly better than a lineal model does. If the results of the LRT were significant, effect estimates were calculated for separate intervals of DAL, using the knots defined in the cubic splines as cut-offs.

To assess the influence of sex, kidney function and dietary fibres on the relation between DAL, TBS and BMD, we tested for interaction by adding the product term of each DAL variable with sex, eGFR or dietary fibre plus eGFR or dietary fibre as independent variables to our model 2 . Only if the $P$ for interaction was $<0.10$, results were stratified according to the population median of eGFR or dietary fibre intake.

We performed several sensitivity analyses. We re-ran our main analyses in model 2 after exclusion of participants with incomplete dietary intake data. Moreover, we excluded participants with type 2 diabetes mellitus because incidence has been shown to be higher in people with high DAL [24] and diabetes patients tend to have lower TBS $[25,26]$. We used the multiple imputation procedure to deal with missing covariates (details in S-Tables 1 and 2). All analyses were performed 
using SPSS (version 22, IBM Corp, New York, United States of America) and R (Version 0.99.484-2009-2015 RStudio Inc., Vienna, Austria) statistical software. A $p$ value of 0.05 was considered to be significant.

\section{Results}

\section{Study population}

Overall, characteristics of our study population did not markedly differ between the three cohorts of the Rotterdam study (Table 1). However, by study design, participants in the third cohort were younger (median $(\mathrm{IQR}=57(52,60)$ versus 71 $(69,75)$ in the second and $78(75,82)$ years in the first cohort). Moreover, these younger participants were more likely to have higher total energy intakes, be less physically active and to have better renal function (Table 1). Whereas the majority of females in the first and second cohort ( $>90 \%)$ were postmenopausal, this was only $77 \%$ in the third cohort. The median spinal TBS was consistent across cohorts, but younger participants were more likely to have higher BMD (Table 1). NEAP was significantly correlated with AnPro/K (Pearson's $r=0.40$ ) and to VegPro/K (Pearson's $r=0.23$ ). However, the two different ratios of protein to potassium were not significantly correlated to each other. Moreover, NEAP and the AnPro/K were negatively correlated with dietary fibre intake (Pearson's $r=-0.47$ and -0.30 ), whereas fibre was weakly positively correlated to the VegPro/K (Pearson's $r=0.13, P$ for all significant correlations $<0.001)$. An overview of food groups explaining most of the variance in NEAP, VegPro/K and AnPro/K is shown in S-Table 3.

\section{Associations between DAL, TBS and BMD}

High NEAP was significantly associated with low TBS in our basic models ( $\beta=-0.04,95 \% \mathrm{CI}=-0.08,-0.01$ ) (Table 2 , model 1). Also, the ratios of vegetable protein and animal protein to potassium were significantly associated with TBS, but in opposite directions. Whereas high VegPro/K was associated with high TBS $(\beta=0.06$ (95\% CI $=0.04,0.07)$, high AnPro/K was associated with low TBS $(\beta=-0.07$ (95\% $\mathrm{CI}=-0.10,-0.04)$. These associations were independent of confounders (Table 2, model 2). For both protein to potassium ratios, LRT indicated the presence of a non-linear relationship, of which are visualized in Fig. 1. Positive associations between VegPro/K and TBS reached a plateau at the population mean VegPro/K $(\approx 0.25 \mathrm{~g} / \mathrm{mEq})$. In contrast, negative associations between AnPro/K and TBS become prominent at the population mean $(\approx 0.34 \mathrm{~g} / \mathrm{mEq})$ only. No significant associations between any of our DAL measures and BMD were observed in any of our models (Table 2), nor were there any indications for non-linearity of these associations.

\section{Additional analyses}

We observed a potential interaction between renal function and VegPro/K in relation to BMD ( $\mathrm{P}$ for interaction $=0.06$ ). Stratified analyses suggested that VegPro/K might be associated with low BMD in subjects with renal function below the median $(\beta=-0.05,95 \% \mathrm{CI}=-0.15,0.05)$ but not in subjects with renal function $\geq$ the median $(\beta=0.01,95 \% \mathrm{CI}=-0.03$, $0.04)$. No evidence for other interactions between DAL and renal function in relation to TBS or BMD were observed (P for interaction all $>0.16$ ).

Moreover, we observed evidence for a potential interaction between NEAP and dietary fibre intake in relation to TBS $(P$ for interaction $=0.06)$ and $\mathrm{BMD}(P$ for interaction $<0.01)$. Stratified analyses were suggestive for a stronger association between NEAP and low TBS in participants with high fibre intake $((\beta-0.03 ; 95 \% \mathrm{CI}-0.08,0.02)$ than in those with low fibre intake $((\beta-0.01 ; 95 \% \mathrm{CI}-0.05,0.03$, S-Table $4 \mathrm{a})$. Also, data suggested that NEAP was associated with low BMD in subjects with high fibre intake only $(\beta(95 \% \mathrm{CI})=-0.03$ $(-0.08,-0.02))$. No other interactions between DAL and dietary fibre in relation to TBS or BMD were observed (STable 4b). Analyses in subgroups with complete FFQ data $(n=3170)$ or without type 2 diabetes $(n=4696)$ showed similar results as our main analyses (S-Table 5).

\section{Discussion}

\section{Summary of our main findings and comparisons to other studies}

In our population of middle-aged and elderly participants, we observed a negative association between NEAP and trabecular bone integrity reflected by the novel trabecular bone score. This negative association was also observed for a high AnPro/K but not for a VegPro/K.

In contrast, we observed no significant association between DAL and BMD. Although both TBS and BMD are derived from the same DXA images, they do reflect different characteristics of the bone. With BMD, the degree of mineralization is quantified, whereas TBS rather combines information on connectivity density, trabecular separation and trabecular number [2]. The results of our main analyses indicate that DAL (NEAP and AnPro/K) might be detrimental to bone health by influencing the trabecular integrity, without necessarily altering BMD.

To our knowledge, we are the first to study associations between DAL and TBS. However, treatment with K-citrate with the aim to neutralize dietary acid load for 24 months was shown to improve bone micro-architecture, reflected by trabecular thickness and number in 201 elderly [27]. Associations between DAL and BMD have been studied 
Table 1 Characteristics in three cohorts of the Rotterdam study (RS), total $n=4672$

\begin{tabular}{|c|c|c|c|c|c|c|}
\hline \multirow[b]{3}{*}{ Age $(\text { year })^{\mathrm{a}}$} & \multirow{2}{*}{\multicolumn{2}{|c|}{$\begin{array}{l}\text { First cohort of the RS } \\
N=1229\end{array}$}} & \multirow{2}{*}{\multicolumn{2}{|c|}{$\begin{array}{l}\text { Second cohort of the } \\
\text { RS } \\
N=1440\end{array}$}} & \multirow{2}{*}{\multicolumn{2}{|c|}{$\begin{array}{l}\text { Third cohort of the } \\
\text { RS } \\
N=2003\end{array}$}} \\
\hline & & & & & & \\
\hline & 78 & $(75,82)$ & 71 & $(69,73)$ & 57 & $(52,60)$ \\
\hline Height $(\mathrm{cm})^{\mathrm{a}}$ & 165 & $(159,173)$ & 167 & $(161,175)$ & 170 & $(164,178)$ \\
\hline Weight $(\mathrm{kg})^{\mathrm{a}}$ & 74 & $(66,83)$ & 77 & $(68,86)$ & 78 & $(69,87)$ \\
\hline Spinal TBS & 1.29 & $(1.22,1.36)$ & 1.30 & $(1.23,1.36)$ & 1.30 & $(1.21,1.37)$ \\
\hline Spinal BMD (g/cm²) & 1.11 & $(0.97,1.27)$ & 1.12 & $(0.98,1.28)$ & 1.15 & $(1.02,1.30)$ \\
\hline Total energy intake $(\mathrm{kcal} / \mathrm{day})^{\mathrm{a}}$ & 1934 & $(1549,2387)$ & 1955 & $(1559,2369)$ & 2233 & $(1852,2720)$ \\
\hline Plasma vitamin D (nmol/l/) & 54 & $(37,73)$ & 62 & $(43,84)$ & 61 & $(43,82)$ \\
\hline NEAP $(g / m E q)$ & 35 & $(28,42)$ & 35 & $(29,42)$ & 36 & $(30,43)$ \\
\hline VegPro/K $(\mathrm{g} / \mathrm{mEq}$ & 0.23 & $(0.19,0.28)$ & 0.24 & $(0.19,0.28)$ & 0.26 & $(0.21,0.31)$ \\
\hline AnPro/K (g/mEq) & 0.33 & $(0.25,0.43)$ & 0.33 & $(0.25,0.43)$ & 0.33 & $(0.25,0.43)$ \\
\hline Physical activity (METh/week) & 90 & $(64,123)$ & 81 & $(55,108)$ & 43 & $(18,81)$ \\
\hline eGFRcrcys (ml/min) & 65 & $(56,76)$ & 74 & $(64,83)$ & 87 & $(78,95)$ \\
\hline Sex (\% males) & 42 & & 44 & & 42 & \\
\hline \multicolumn{7}{|l|}{ Education $(\%)$} \\
\hline Low & \multicolumn{2}{|l|}{9} & \multicolumn{2}{|l|}{28} & \multicolumn{2}{|l|}{25} \\
\hline Middle & \multicolumn{2}{|l|}{12} & \multicolumn{2}{|l|}{20} & \multicolumn{2}{|l|}{20} \\
\hline Middle-high & \multicolumn{2}{|l|}{16} & \multicolumn{2}{|l|}{25} & \multicolumn{2}{|l|}{21} \\
\hline High & \multicolumn{2}{|l|}{63} & \multicolumn{2}{|l|}{24} & \multicolumn{2}{|l|}{35} \\
\hline \multicolumn{7}{|l|}{ Income $(\%)$} \\
\hline Low & \multicolumn{2}{|l|}{64} & \multicolumn{2}{|l|}{38} & \multicolumn{2}{|l|}{23} \\
\hline Middle & \multicolumn{2}{|l|}{28} & \multicolumn{2}{|l|}{63} & \multicolumn{2}{|l|}{24} \\
\hline Middle-high & \multicolumn{2}{|l|}{3} & \multicolumn{2}{|l|}{12} & \multicolumn{2}{|l|}{13} \\
\hline High & \multicolumn{2}{|l|}{5} & \multicolumn{2}{|l|}{15} & \multicolumn{2}{|l|}{40} \\
\hline Current smokers (\%) & 7 & & 10 & & 13 & \\
\hline Type 2 diabetes (\%) & 12 & & 12 & & 6 & \\
\hline $\begin{array}{l}\text { Menopausal status } \\
\quad(\% \text { postmenopausal })^{\mathrm{b}}\end{array}$ & 97 & & 92 & & 77 & \\
\hline Use of any dietary supplement $(\%)^{\mathrm{C}}$ & 60 & & 59 & & 60 & \\
\hline Use of lipid lowering drugs & 34 & & 32 & & 27 & \\
\hline Use of antihypertensives ${ }^{\mathrm{d}}$ & 23 & & 17 & & 6 & \\
\hline
\end{tabular}

$B M D$ bone mineral density, $B M I$ body mass index, HRT Hormone replacement therapy, METh metabolic equivalent of tasks in hours, TBS trabecular bone score, $d P R A L$ dietary potential renal acid load, TPro/K total protein/ potassium ratio, VegPro/K Vegetable protein/potassium ratio, AnPro/K animal protein/potassium ratio

${ }^{\text {a }}$ Median (interquartile range)

${ }^{\mathrm{b}}$ Applicable to females only

${ }^{\mathrm{c}}$ Use of any dietary supplement $\geq 1$ time/month

${ }^{\mathrm{d}}$ Including diuretics

extensively with conflicting results $[9,28-30]$, which could be in part explained by methodological differences between studies. Protein intake is an important contributor to DAL. A recent meta-analyses on 12 prospective cohort studies showed that total dietary protein consumption may decrease the risk of hip fracture but concluded that evidence was insufficient to draw the same conclusion for animal or vegetable protein. No significant overall associations were observed of total, animal or vegetable protein in relation to all fractures and limb fractures in this study, and the lack of studies on vertebral fractures was emphasized by the authors [31].

\section{Potential non-linearity of the associations}

Moreover, our results suggest that the shape of associations of the VegPro/K and AnPro/K with TBS are non-linear. Whereas the negative associations are only observed at the highest AnPro/K, the positive associations were only observed at the lowest VegPro/K only. This difference might be due to the 
Table 2 Linear associations between DAL, TBS and BMD

\begin{tabular}{|c|c|c|c|c|}
\hline & \multicolumn{2}{|c|}{ Model 1} & \multicolumn{2}{|c|}{ Model 2} \\
\hline & \multicolumn{2}{|l|}{ Basic } & \multicolumn{2}{|c|}{ Confounders } \\
\hline & $\beta$ & $95 \% \mathrm{CI}$ & $\beta$ & $95 \% \mathrm{CI}$ \\
\hline & \multicolumn{4}{|c|}{ Trabecular bone score } \\
\hline NEAP & -0.04 & $(-0.08,-0.01)$ & -0.04 & $(-0.07,-0.01)$ \\
\hline VegPro/K & 0.06 & $(0.04,0.07)^{\mathrm{a}}$ & 0.05 & $(0.01,0.08)$ \\
\hline \multirow[t]{2}{*}{ AnPro/K } & -0.07 & $(-0.10,-0.04)^{\mathrm{a}}$ & -0.08 & $(-0.11,-0.04)$ \\
\hline & \multicolumn{4}{|c|}{ Bone mineral density } \\
\hline NEAP & -0.02 & $(-0.05,0.01)$ & -0.02 & $(-0.05,0.01)$ \\
\hline VegPro/K & -0.01 & $(-0.04,0.01)$ & -0.00 & $(-0.04,0.03)$ \\
\hline AnPro/K & -0.02 & $(-0.03,0.04)$ & -0.02 & $(-0.05,0.02)$ \\
\hline
\end{tabular}

Regression coefficients represent changes in Z-scores of BMD or TBS for each Z-score increase in DAL. Model 1: Adjusted for age, sex, total energy intake, body weight and height and Rotterdam study cohort. Model 2: Model $1+$ education and smoking, dietary calcium intake and alcohol consumption. Significant associations $(P$ value $<0.05)$ in bold

${ }^{a}$ Presence of a non-linear relationship, based on a likelihood ratio test comparing the linear model to a non-linear model

difference in sulphur containing amino acids [32], since animal proteins may contain more sulphur (e.g. methionine and cysteine) and therefore produce more acid than do vegetable proteins. It has been noted that animal protein by itself, independent of the ratio to potassium, was beneficially associated with BMD and fracture risk in two other epidemiological studies [33, 34]. The non-linear shape of the association between VegPro/K and TBS in our population might reflect the importance of the anabolic effects of protein at acid loads below the mean, since amino acids are important substrates for building bone matrix [11]. Moreover, DAL has been suggested to affect bone outcomes via increased calcium excretion by the kidneys since bone minerals (mainly calcium) can be used as a base to neutralize low blood pH [35]. However, Cao and Nielsen recently concluded that, although a diet with a high acid load due to high intakes of meat and protein might increase renal acid load and urinary calcium excretion, demineralized bone was not necessarily the source of this extra calcium lost in urine. In contrast, the authors suggest that in case of diet-induced renal calcium loss, the body will compensate by promoting intestinal calcium uptake as primary mechanism to neutralize low blood $\mathrm{pH}$ [35].

\section{DAL: only adversely associated with bone outcomes in subgroups?}

Since the kidneys are the primary organs to regulate chronic systemic acidosis, it could be argued that high DAL might be detrimental only in specific subgroups, such as those with low renal function.

We observed no significant interaction between DAL and renal function in relation to bone outcomes in our cohort of Dutch elderly. However, data suggest that a negative association between $\mathrm{VegPro} / \mathrm{K}$ and BMD might be present only in subjects with a renal function below the population median. Also, in a cohort of Swedish elderly, stratified analyses based on renal function using a more stringent cut-off of $60 \mathrm{ml} / \mathrm{min}$ showed no associations between DAL and risk of fractures (from the neck down) in both strata [9]. Some studies have suggested that the potential adverse effects of DAL on bone outcomes might be present only in subjects with the lowest intake of alkali-forming nutrients. For example, an inverse association between dietary potential renal acid load (dPRAL, another measure of DAL in which the intake of phosphorus is taken into account) and proximal femur BMD was detected among men with low dietary calcium intake ( $<800 \mathrm{mg} /$ day) only [28]. Traditionally, the Dutch diet is rich in dairy products and calcium [36], which is also reflected by the mean of $1010 \mathrm{mg}$ of daily calcium intake in our population. Therefore, we were unable to study the association between DAL and bone outcomes in participants with low calcium intake in our population. In addition to interaction between DAL and acid-forming or alkali-forming nutrients, we hypothesised that interaction between DAL and other nutrients in the overall diet might occur. More specifically and as suggested by Cao et al. previously [15], dietary fibre might inhibit intestinal absorption of dietary calcium and dietary acid
Fig. 1 Non-linear associations between ratios of vegetable or animal protein to potassium and spinal trabecular bone score, reflecting trabecular bone integrity, using basic models adjusted for age, sex, body weight and height and total energy intake
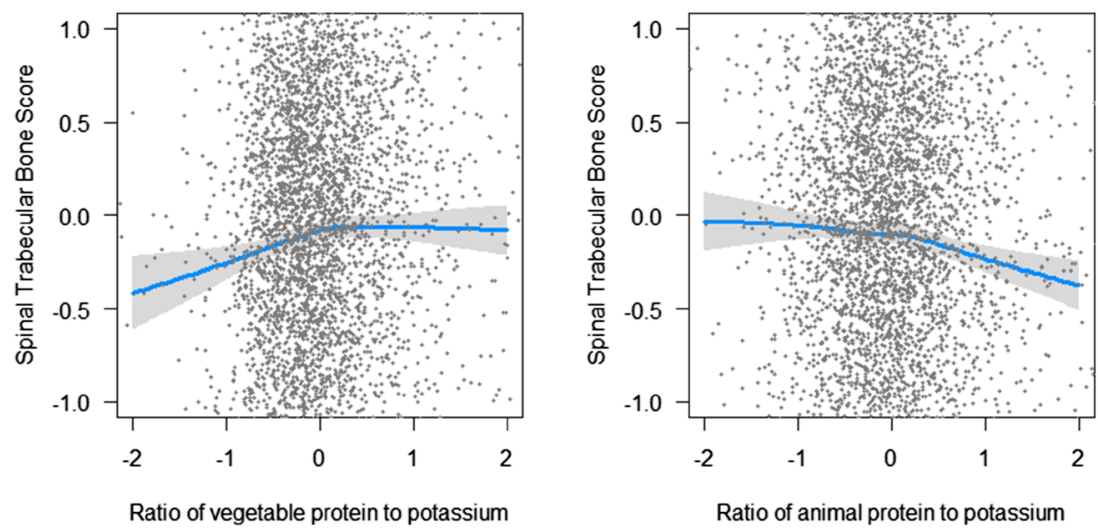
load might reduce uptake of calcium from the bloodstream by the bones. DAL might therefore be more strongly associated with unfavourable bone outcomes in subgroups with high fibre intake. Indeed, our data were suggestive for an interaction between NEAP and dietary fibre in relation to BMD and TBS. Dietary fibre derived from grains might present in high DAL diets and derived from fruits and vegetables in low DAL diets. Altogether, these findings might imply that DAL might be adversely associated with bone outcomes in subgroups only, and that the food groups that determine DAL in a specific population (e.g. grains, fruits and vegetables) matter.

\section{Strengths and limitations}

Our study has several strengths. To our knowledge, we were the first to investigate DAL in relation to trabecular integrity of the bone in a large population-based study. Moreover, we have used different measures of DAL, allowing us to separately investigate which components of the DAL might be important for bone. However, we also recognize some limitations. The use of an FFQ to assess dietary intake is prone to measurement error. To account for systematic measurement error, we adjusted our analyses for total energy intake using the residual method [20]. Also, we performed sensitivity analyses to account for incomplete dietary intake data. Results from our sensitivity analysis showed similar effect sizes before and after exclusion of participants with incomplete data. Unfortunately, we did not have biomarkers of acid load, such as urinary $\mathrm{pH}$ or serum bicarbonate levels to validate our findings. Random error might have still been present and diluted our associations. TBS data were only available in a subsample of the Rotterdam study. However, characteristics of the participants included in our study did not markedly differ from those included in the full third cohort of the Rotterdam study. Hence, we believe that our results are valid for our full cohort. Due to the limited follow-up period, we were not able to study associations between DAL and risk of fractures yet. Lastly, we did not have sufficient participants with an eGFR $<60 \mathrm{ml} / \mathrm{min}$ to study the influence of impaired versus normal renal function, limiting any conclusions on specific associations in those with impaired renal function or renal failure.

\section{Practical implications and recommendations for future research}

Our results do not support the hypothesis that high DAL is associated with low BMD. In contrast, we observed significant associations with low TBS. Moreover, they are suggestive for differently shaped, non-linear associations between AnPro/K and VegPro/K with TBS. Future studies are needed to confirm our results in other populations. Also, future studies may include a longitudinal approach in which associations between DAL and changes in BMD or TBS and subsequent risk of fragility fractures could be evaluated. Moreover, our population had low median DAL and small variance compared to other studies $[32,37]$. Future studies in populations with more extreme ranges of DAL might provide additional insights. Lastly, we hypothesised that associations with TBS might add to the explanation of conflicting results on DAL and fracture risk. Long-term studies will be needed to confirm this hypothesis. Due to its contribution to high DAL, protein has been suggested to adversely affect skeletal health [7, 35]. However, when combined with exercise, protein intake, an important component of the acid load variables in our study, also has positive musculoskeletal effects, for example for the prevention of sarcopenia (loss of muscle mass) [38, 39]. Therefore, one can argue that recommendations to reduce animal protein intake to facilitate a reduction in DAL in the ageing population might be undesired. In contrast, a proper balance between protein from animal sources by protein from vegetable sources might be beneficial for overall musculoskeletal health. Future research would be needed to further clarify the role of protein sources in skeletal health.

\section{Conclusions}

In our population of middle-aged and elderly, high NEAP was associated with low trabecular bone integrity. Associations of AnPro/K and VegPro/K and TBS were non-linear and differently shaped.

No significant associations with BMD were observed, nor was any interaction between DAL and renal function in relation to TBS or BMD. Only in participants with high intake of dietary fibre, NEAP might be detrimental to bone outcomes. These findings imply that nutrients that characterise a high DAL diet but are not incorporated in the DAL equation might influence associations of DAL with TBS and BMD.

Acknowledgements Oscar H. Franco, Jessica C. Kiefte-de Jong and Ester A.L. de Jonge work in ErasmusAGE, a centre for ageing research across the life course funded by Nestlé Nutrition (Nestec Ltd.) and Metagenics Inc. Nestlé Nutrition (Nestec Ltd.) and Metagenics Inc. had no role in the design and conduct of the study; collection, management, analysis and interpretation of the data and preparation, review or approval of the manuscript. Dr. Fernando Rivadeneira received a grant from the Netherlands Organization for Scientific Research (NWO, VIDI 016.136.367). Ester A.L. de Jonge is supported by a grant from the NWO for the graduate program of 2010 (project number: 022.002.023).

The Rotterdam study is supported by the Erasmus MC University Medical Centre and Erasmus University Rotterdam; the Netherlands Organization for Scientific Research (NWO), the Netherlands Organization for Health Research and Development (ZonMw); the Research Institute for Diseases in the Elderly (RIDE); the Netherlands Genomics Initiative (NGI); the Netherlands Consortium of Healthy ageing (NCHA); the Ministry of Education, Culture and Science; the Ministry of Health, Welfare and Sports; the European Commission (DG XII) and the Municipality of Rotterdam. The contribution of inhabitants, general practitioners and pharmacists of the Ommoord district to the Rotterdam study is gratefully acknowledged. 


\section{Compliance with ethical standards}

Ethical approval The Rotterdam study has been approved by the Medical Ethics Committee of the Erasmus MC and by the Ministry of Health, Welfare and Sport of the Netherlands, implementing the Wet Bevolkingsonderzoek: ERGO (Population Studies Act: Rotterdam Study). All participants provided written informed consent to participate in the study and to obtain information from their treating physicians. All procedures performed in studies involving human participants were in accordance with the ethical standards of the institutional and/or national research committee and with the 1964 Helsinki Declaration and its later amendments or comparable ethical standards.

\section{Conflicts of interest None.}

Open Access This article is distributed under the terms of the Creative Commons Attribution-NonCommercial 4.0 International License (http:// creativecommons.org/licenses/by-nc/4.0/), which permits any noncommercial use, distribution, and reproduction in any medium, provided you give appropriate credit to the original author(s) and the source, provide a link to the Creative Commons license, and indicate if changes were made.

\section{References}

1. Caliri A, De Filippis L, Bagnato GL, Bagnato GF (2007) Osteoporotic fractures: mortality and quality of life. Panminerva Med 49(1):21-27

2. Hans D, Barthe N, Boutroy S, Pothuaud L, Winzenrieth R, Krieg MA (2011) Correlations between trabecular bone score, measured using anteroposterior dual-energy X-ray absorptiometry acquisition, and 3-dimensional parameters of bone microarchitecture: an experimental study on human cadaver vertebrae. J Clin Densitom 14(3):302-312. doi:10.1016/j.jocd.2011.05.005

3. Hans D, Goertzen AL, Krieg MA, Leslie WD (2011) Bone microarchitecture assessed by TBS predicts osteoporotic fractures independent of bone density: the Manitoba study. J Bone Miner Res 26(11):2762-2769. doi:10.1002/jbmr.499

4. Atanasovska B, Oei, L; Hofman, A; Aubry-Rozier, B; Uitterlinden, A.G; Zillikens, M.C; Oei, E.H.G; Hans, D; Rivadeneira, F. Prediction of vertebral fracture by trabecular bone score in elderly women of the Rotterdam study: https://www.slideshare.net/ stichtingiwo/erasmus-31983331

5. Frassetto LA, Todd KM, Morris RC Jr, Sebastian A (1998) Estimation of net endogenous noncarbonic acid production in humans from diet potassium and protein contents. Am J Clin Nutr 68(3):576-583

6. Hietavala EM, Stout JR, Hulmi JJ, Suominen H, Pitkanen H, Puurtinen R, Selanne H, Kainulainen H, Mero AA (2015) Effect of diet composition on acid-base balance in adolescents, young adults and elderly at rest and during exercise. Eur J Clin Nutr 69(3):399-404. doi:10.1038/ejcn.2014.245

7. Bonjour JP (2013) Nutritional disturbance in acid-base balance and osteoporosis: a hypothesis that disregards the essential homeostatic role of the kidney. Br J Nutr 110(7):1168-1177. doi:10.1017/ s0007114513000962

8. Fenton TR, Tough SC, Lyon AW, Eliasziw M, Hanley DA (2011) Causal assessment of dietary acid load and bone disease: a systematic review \& meta-analysis applying Hill's epidemiologic criteria for causality. Nutr J 10:41. doi:10.1186/1475-2891-10-41

9. Jia T, Byberg L, Lindholm B, Larsson TE, Lind L, Michaelsson K, Carrero JJ (2015) Dietary acid load, kidney function, osteoporosis, and risk of fractures in elderly men and women. Osteoporos Int 26(2):563-570. doi:10.1007/s00198-014-2888-x

10. Dargent-Molina P, Sabia S, Touvier M, Kesse E, Breart G, ClavelChapelon F, Boutron-Ruault MC (2008) Proteins, dietary acid load, and calcium and risk of postmenopausal fractures in the E3N French women prospective study. J Bone Miner Res 23(12): 1915-1922. doi:10.1359/jbmr.080712

11. Sebastian A (2005) Dietary protein content and the diet's net acid load: opposing effects on bone health. Am J Clin Nutr 82(5):921922

12. Gropper SG, Smith JL, Groff JL (2005) Advanced nutrition and human metabolism, chapter 14. Thomson Wadsworth, Belmont

13. Moe S, Drueke T, Cunningham J, Goodman W, Martin K, Olgaard K, Ott S, Sprague S, Lameire N, Eknoyan G, Kidney Disease: Improving Global O (2006) Definition, evaluation, and classification of renal osteodystrophy: a position statement from kidney disease: improving global outcomes (KDIGO). Kidney Int 69(11): 1945-1953. doi:10.1038/sj.ki.5000414

14. Fried LF, Biggs ML, Shlipak MG, Seliger S, Kestenbaum B, Stehman-Breen C, Sarnak M, Siscovick D, Harris T, Cauley J, Newman AB, Robbins J (2007) Association of kidney function with incident hip fracture in older adults. J Am Soc Nephrol 18(1):282-286. doi:10.1681/ASN.2006050546

15. Cao JJ, Johnson LK, Hunt JR (2011) A diet high in meat protein and potential renal acid load increases fractional calcium absorption and urinary calcium excretion without affecting markers of bone resorption or formation in postmenopausal women. J Nutr 141(3): 391-397. doi:10.3945/jn.110.129361

16. Hofman A, Brusselle GG, Darwish Murad S, van Duijn CM, Franco OH, Goedegebure A, Ikram MA, Klaver CC, Nijsten TE, Peeters RP, Stricker BH, Tiemeier HW, Uitterlinden AG, Vernooij MW (2015) The Rotterdam study: 2016 objectives and design update. Eur J Epidemiol 30(8):661-708. doi:10.1007/s10654-0150082-x

17. Goldbohm RA, van den Brandt PA, Brants HA, van't Veer P, Al M, Sturmans F, Hermus RJ (1994) Validation of a dietary questionnaire used in a large-scale prospective cohort study on diet and cancer. Eur J Clin Nutr 48(4):253-265

18. Klipstein-Grobusch K, den Breeijen JH, Goldbohm RA, Geleijnse JM, Hofman A, Grobbee DE, Witteman JCM (1998) Dietary assessment in the elderly: validation of a semiquantitative food frequency questionnaire. Eur J Clin Nutr 52(8):588-596. doi:10.1038/ sj.ejcn.1600611

19. Dutch Food Composition Table (2006) Nutrition Centre

20. Willett WC, Howe GR, Kushi LH (1997) Adjustment for total energy intake in epidemiologic studies. Am J Clin Nutr 65(4 Suppl):1220S-1228S discussion 1229S-1231S

21. Stel VS, Smit JH, Pluijm SM, Visser M, Deeg DJ, Lips P (2004) Comparison of the LASA physical activity questionnaire with a 7 day diary and pedometer. J Clin Epidemiol 57(3):252-258. doi:10. 1016/j.jclinepi.2003.07.008

22. Harlow SD, Gass M, Hall JE, Lobo R, Maki P, Rebar RW, Sherman S, Sluss PM, de Villiers TJ, Group SC (2012) Executive summary of the stages of reproductive aging workshop +10 : addressing the unfinished agenda of staging reproductive aging. Menopause 19(4): 387-395. doi:10.1097/gme.0b013e31824d8f40

23. de Jonge EA, Kiefte-de Jong JC, de Groot LC, Voortman T, Schoufour JD, Zillikens MC, Hofman A, Uitterlinden AG, Franco $\mathrm{OH}$, Rivadeneira F (2015) Development of a food group-based diet score and its association with bone mineral density in the elderly: the Rotterdam study. Nutrients 7(8):6974-6990. doi:10.3390/ nu7085317

24. Fagherazzi G, Vilier A, Bonnet F, Lajous M, Balkau B, BoutronRualt MC, Clavel-Chapelon F (2014) Dietary acid load and risk of type 2 diabetes: the E3N-EPIC cohort study. Diabetologia 57(2): 313-320. doi:10.1007/s00125-013-3100-0 
25. Oei L, Rivadeneira F, Zillikens MC, Oei EH (2015) Diabetes, diabetic complications, and fracture risk. Curr Osteoporos Rep 13(2): 106-115. doi:10.1007/s11914-015-0260-5

26. Leslie WD, Aubry-Rozier B, Lamy O, Hans D, Manitoba Bone Density P (2013) TBS (trabecular bone score) and diabetesrelated fracture risk. J Clin Endocrinol Metab 98(2):602-609. doi: $10.1210 /$ jc. $2012-3118$

27. Jehle S, Hulter HN, Krapf R (2013) Effect of potassium citrate on bone density, microarchitecture, and fracture risk in healthy older adults without osteoporosis: a randomized controlled trial. J Clin Endocrinol Metab 98(1):207-217. doi:10.1210/jc.2012-3099

28. Mangano KM, Walsh SJ, Kenny AM, Insogna KL, Kerstetter JE (2014) Dietary acid load is associated with lower bone mineral density in men with low intake of dietary calcium. J Bone Miner Res 29(2):500-506. doi:10.1002/jbmr.2053

29. McLean RR, Qiao N, Broe KE, Tucker KL, Casey V, Cupples LA, Kiel DP, Hannan MT (2011) Dietary acid load is not associated with lower bone mineral density except in older men. J Nutr 141(4):588594. doi:10.3945/jn.110.135806

30. Macdonald HM, New SA, Fraser WD, Campbell MK, Reid DM (2005) Low dietary potassium intakes and high dietary estimates of net endogenous acid production are associated with low bone mineral density in premenopausal women and increased markers of bone resorption in postmenopausal women. Am J Clin Nutr 81(4):923-933

31. Wu AM, Sun XL, Lv QB, Zhou Y, Xia DD, Xu HZ, Huang QS, Chi YL (2015) The relationship between dietary protein consumption and risk of fracture: a subgroup and dose-response meta-analysis of prospective cohort studies. Scientific reports 5:9151. doi:10.1038/ srep09151

32. Zwart SR, Hargens AR, Smith SM (2004) The ratio of animal protein intake to potassium intake is a predictor of bone resorption in space flight analogues and in ambulatory subjects. Am J Clin Nutr 80(4):1058-1065
33. Langsetmo L, Barr SI, Berger C, Kreiger N, Rahme E, Adachi JD, Papaioannou A, Kaiser SM, Prior JC, Hanley DA, Kovacs CS, Josse RG, Goltzman D, CaMos Research G (2015) Associations of protein intake and protein source with bone mineral density and fracture risk: a population-based cohort study. J Nutr Health Aging 19(8):861-868. doi:10.1007/s12603-015-0544-6

34. Langsetmo L, Shikany JM, Cawthon PM, Cauley JA, Taylor BC, Vo TN, Bauer DC, Orwoll ES, Schousboe JT, Ensrud KE, Osteoporotic Fractures in Men Research G (2017) The association between protein intake by source and osteoporotic fracture in older men: a prospective cohort study. J Bone Miner Res 32(3):592-600. doi:10.1002/jbmr.3058

35. Cao JJ, Nielsen FH (2010) Acid diet (high-meat protein) effects on calcium metabolism and bone health. Curr Opin Clin Nutr Metab Care 13(6):698-702. doi:10.1097/MCO.0b013e32833df691

36. van den Berg P, van Haard PM, van den Bergh JP, Niesten DD, van der Elst M, Schweitzer DH (2014) First quantification of calcium intake from calcium-dense dairy products in Dutch fracture patients (the Delft cohort study). Nutrients 6(6):2404-2418. doi:10.3390/ nu6062404

37. Luis D, Huang X, Riserus U, Sjogren P, Lindholm B, Arnlov J, Cederholm T, Carrero JJ (2015) Estimated dietary acid load is not associated with blood pressure or hypertension incidence in men who are approximately 70 years old. J Nutr 145(2):315-321. doi: 10.3945/jn.114.197020

38. Wall BT, Cermak NM, van Loon LJ (2014) Dietary protein considerations to support active aging. Sports Med 44(Suppl 2):S185$\mathrm{S} 194$

39. Cermak NM, Res PT, de Groot LC, Saris WH, van Loon LJ (2012) Protein supplementation augments the adaptive response of skeletal muscle to resistance-type exercise training: a meta-analysis. Am J Clin Nutr 96(6):1454-1464 